\title{
Electrochemically Directed Modification of ITO Electrodes and Its Feasibility for the Immunosensor Development
}

\author{
Daegeun Yu and Kyuwon Kim*
Department of Chemistry, Lniversity of Incheon, Incheon $+02-749$, Korea. ${ }^{*}$ E-mail: kutn'on_kimaincheon ack kr Received Februarv 3, 2009. Accepted Februarv 23. 2009

Key Wonds: Electrochemical modification. Indium-tin-oxide. Fluorescence. Immunosensonor. Immunoassaỵ

During the last several decades, indium-tin-oxide (ITO) films on glass or quartz substrates have been increasingly employed as an electrode surface because it has prominent characteristics such as a good electrical conductivity. wide electrochemical working window. and high optical transparency. 'The films are commonly deposited on surfaces by e-beam evaporation. physical vapor deposition. or sputtering method. ${ }^{2}$ Main use of ITO is to make transparent conductive films for displays. solar cells. antistatic coatings and biosensors. $^{3-6}$ Numerous studies have been reported on the development of biosensors on ITO electrodes utilizing electronic and optical properties for direct electron transfer of proteins, glucose sensors. electrochemical nucleic acid biosensors. microfluidic on-chip detection. and electrochemiluminescence analysis. ${ }^{\nabla-10}$

Biosensors application requires chemical modification of ITO electrode surfaces with organic anchor molecules. In most previous reports for the application, $\omega$-functionalized alky lsilane such as amino- or mercapto-propyltrialkoxysilane have been used as anchor molecules that are attached on the hydroxylated ITO surface through self-assembly process of silane part. For the immobilization of biomolecules or their ligands, the end part of the silane molecules should be activated by chemical coupling reaction with homo- or heterobifunctional cross-linker molecules that contain biomoleculereactive group such as 1 -hydroxy succinimide (NHS) ester. ${ }^{\text {] }}$ Other modification methods include the formation of organic lay'ers with carboxỵlic and phosphonic acids. and amines. ${ }^{12.14}$ Thorp et al have modified ITO with dicarboxy lic acid for the immobilization of DNA. "Yang and coworkers took advantage of the surface attachment of phosphonic acids onto ITO instead of $(0)$-functionalized silane. ${ }^{16}$ Although those chemical attachments are attractive approaches for the modification of ITO, they are time consuming and require frequently further modification of the attached surface with functional molecules.

Electrochemically directed attachment of various molecules on bare ITO surfaces with organic molecules has demonstrated that it is very rapid and facilitates the precise positioning of adsorbent molecules. ${ }^{17.18}$ Especially. primary amine attachment might be very useful because many functional molecules used in biosensor applications have primary amine. ${ }^{19+9}$ To the best of our knowledge. direct electrochenical modification of bare surface of ITO with primary amine groups has never been reported

In this paper. we report electrochemically directed and addressed modification of ITO electrodes with a biotin-deri- vatized printary anine (BDA) as a model molecule of primary amine and spatially selective immobilization of avidin on the modified surfaces. We also demonstrate the feasibility of the BDA-modified surfaces to a sandwich-type immunosensor using fluorescence detection. Our method is time saving compared to the silanization process because electrochemical reaction for the attachment is completed within several tens of seconds and further modifications are less required. which are
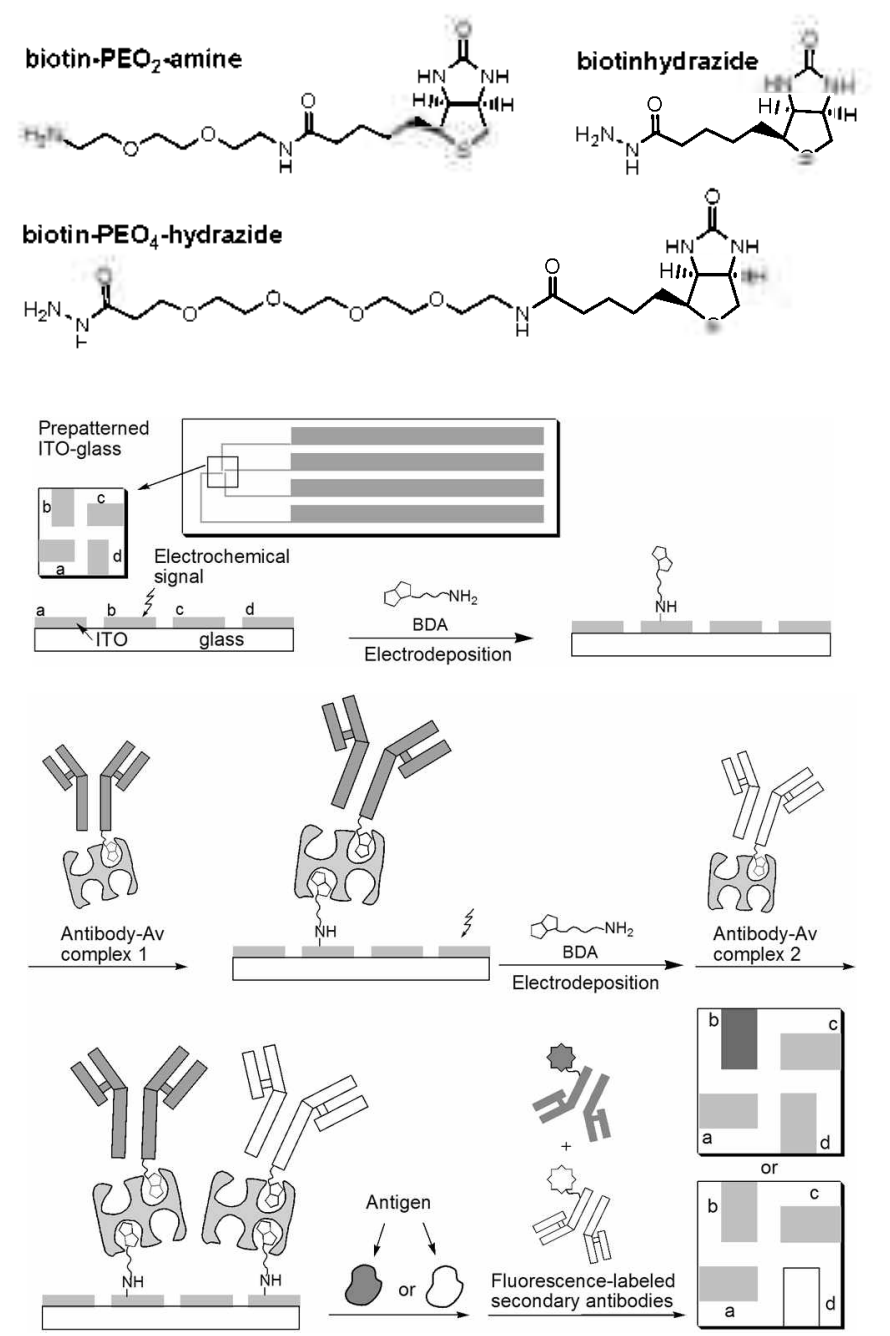

Scheme 1. The chemical structures of BDAs employed in this study and a proposed scheme for micropattening of antibodies and detection of antigens through electrochenically directed modification of bare ITO surfaces with BDAs. 
also important points for the surface preparation of multi-probes biosensor without cross contamination.

\section{Results and Discussion}

Scheme 1 displays overall process of our study including step-by-step modification of ITO electrodes atray with BDA by chemical and electrochemical treatments, a micropatterning of antibody on the modified surface, and a sandwich immunoassay. Individually addressable band-type lTO microelectrodes arrays (IAIM) were prepared on glass substrates by a simple microfabrication process as previously reported. ${ }^{21}$ We employ commercially available three kinds of BDAs: biotin- $\mathrm{PEO}_{2}$-amine (BPA), biotin hydrazide $(\mathrm{BH})$, biotin$\mathrm{PEO}_{4}$-hydrazide $(\mathrm{BPH})$.

To directly immobilize BDAs onto ITO electrode surfaces through the electrochemical reaction, cyclic voltammetry (CV) was performed on a bare ITO electrode as a working electrode pretreated by sequential cleaning with $\mathrm{EtOH}$, acetone, piranha solution, and pure water. Figure 1 shows CV results for three different BDAs solutions with $0.1 \mathrm{M} \mathrm{KCl}$ as an electrolyte, which exhibit similar electrochemical behaviors of irreversible anodic peak over $1.70 \mathrm{~V}$ (vs. $\mathrm{Ag} / \mathrm{AgCl}$ as a reference electrode) on the first cycle and gradual decreases of the current with increasing the number of $\mathrm{CV}$ scan, indicating gradual modification of bare electrode surfaces. Electrolyte only solution and free-biotin solution without primary amine didn't show any noticeable current at the same potential region.

To examine the immobilization of BDAs through the electrochemical manner, the blocking behaviors for the

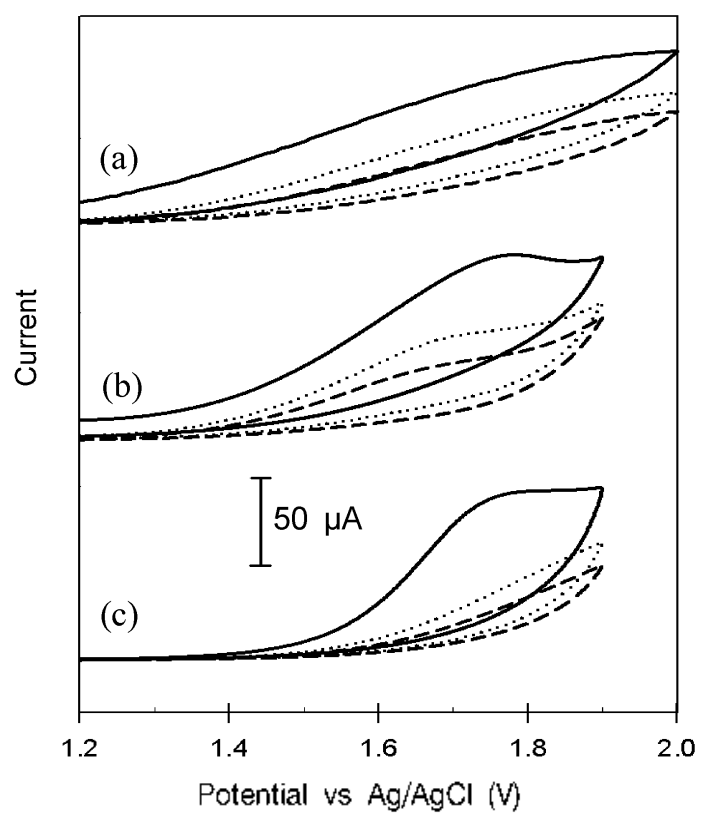

Figure 1. CVs for the electrochemical reactions of three kinds of BDAs ( $10 \mathrm{mM}$ each) on bare ITO surfaces in an aqueous solution with $0.1 \mathrm{M} \mathrm{KCl}$ (all sean rates $=50 \mathrm{~m}$ Visec): Solid line. lirst cycle: dotted line. second cycle: dashed line. third cycle. (ai) biotin hydrazide $(\mathrm{BH})$ (b) biotin-PEO ${ }_{2}$-amine (BPA) (c) biotin-PEO $4^{-}$ hydrazjde (BPH). electron transfer at ITO electrode surfaces after electrochemical oxidation reaction of $\mathrm{BH}$ were investigated by $\mathrm{CV}$ with $\mathrm{Fe}(\mathrm{CN})_{6}{ }^{3-}$ as a redox probe. Figure 2(a) shows that after the electrochemical treatment with $\mathrm{BH}$, the peak separation $\left(\Delta F_{\mathrm{p}}\right)$ on the CV is slightly increased compared to the results for bare ITO surface. $\Delta E_{p}$ for the resulting surface after the electrochemical treatment without $\mathrm{BH}$ was not changed from the value for bare surface. $\Delta \digamma_{p}$ was slightly increased with increasing the concentration of $\mathrm{BH}$ and the number of $\mathrm{CV}$ scan. Figure 2(b) shows that Nyquist plots of faradaic impedance spectroscopic $(\mathrm{FIS})$ results using $\mathrm{Fe}(\mathrm{CN})_{t}{ }^{3}$ as a redox probe support the immobilization of $\mathrm{BH}$. Charge transfer resistance $\left(R_{\mathrm{ct}}\right)$ value from FIS was increased after the electrochemical reaction of $\mathrm{BH}$. All results from the blocking test show that charge transfer rate for the redox reaction of $\mathrm{Fe}(\mathrm{CN})_{6}{ }^{{ }^{-}}$ depends on the electrochemical reaction condition of $\mathrm{BH}$, implying that $\mathrm{BH}$ layer can be formed on ITO surfaces through our method..$^{2-}$ Because the change of $\Delta E_{p \mid}$ and $R_{c t}$ is not significant, the surface coverage of immobilized $\mathrm{BH}$
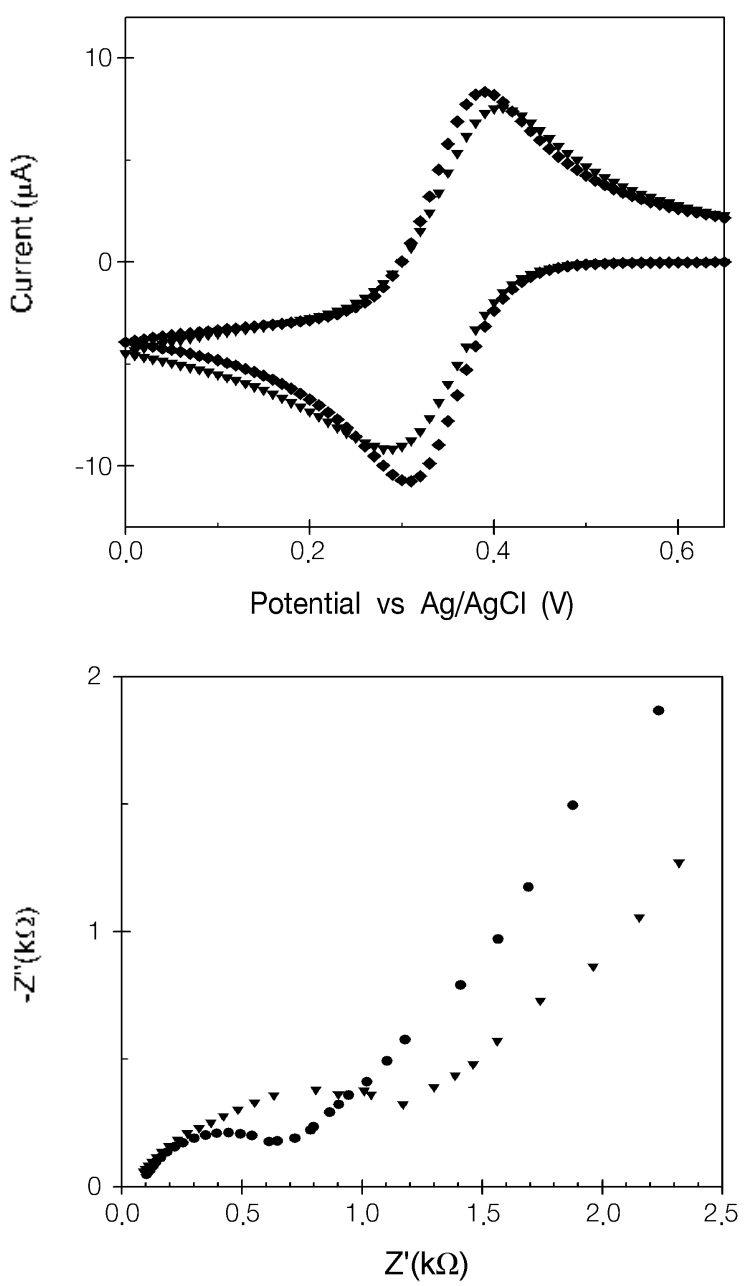

Figure 2. Observation of blocking behaviors from (a) CV (scan rate $=50 \mathrm{mV} / \mathrm{sec}$ ) and (b) FIS results for the electron transtior of $\mathrm{Fe}(\mathrm{C} \mathrm{V}){ }_{6}{ }^{3}$ at ITO clectrode surfáces before $(\diamond)$ and after $(\nabla)$ electrochemical reaction of $\mathrm{BI}$. In the FIS. impedance at the lormal polential ol $\mathrm{Fe}(\mathrm{C} \mathrm{V})_{0}^{3-}$ or $0.35 \mathrm{~V}(\mathrm{~s} . \mathrm{Ag} / \mathrm{AgCl})$ that was superimposed on $50 \mathrm{mV}$ rms sinusoidal potential modulations. was measured for 36 frequencics from $5 \mathrm{kHz}$ to $0.5 \mathrm{~Hz}$. 

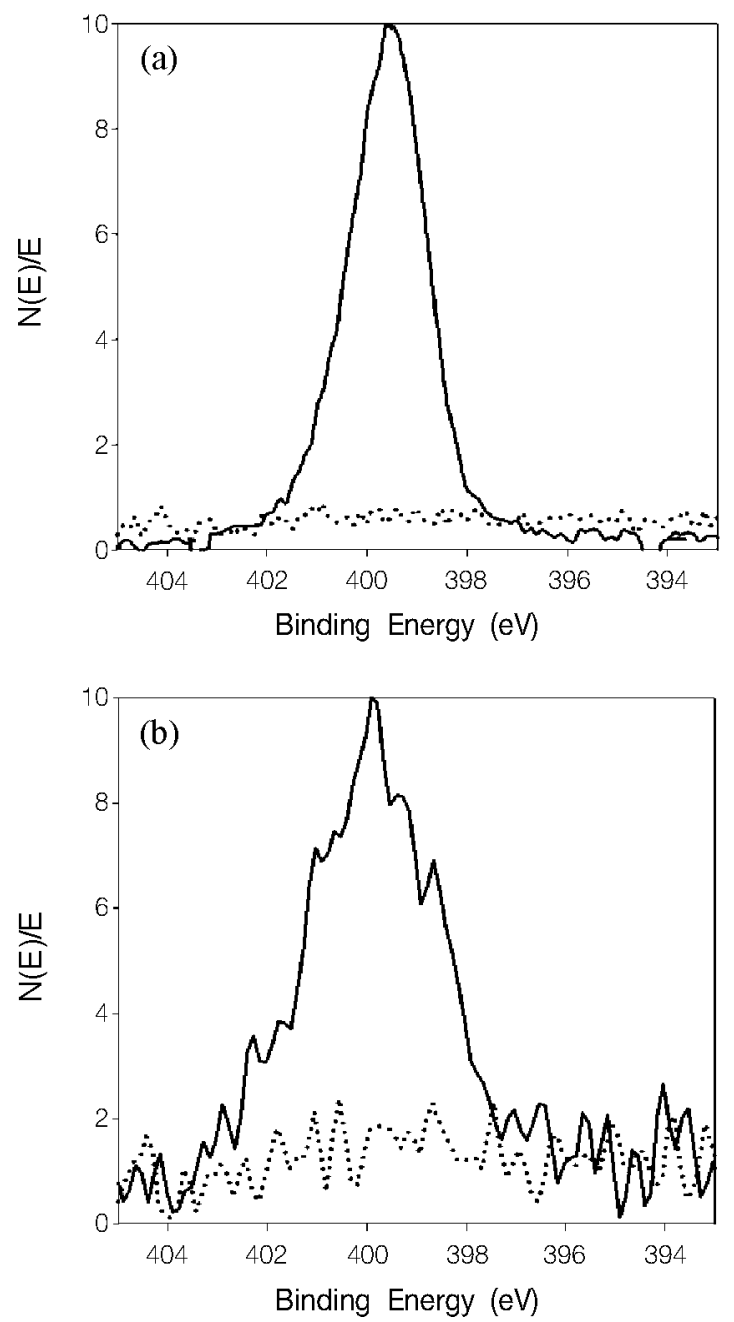

Figure 3. Nomalized XPS results of N( ls) for ITO surfaces before (dashed line) and after ( solid line) electrochemical treatments with (a) $\mathrm{BH}$ and (b) $\mathrm{BPA}$.

might have low value

ITO surfaces before and after electrochemical treatments with BDAs were analyzed by X-ray photoelectron spectroscopy (XPS). As shown in Figure 3. while a N(Is) peak in XPS was not detected for the untreated surfaces. the peak was found at near $400 \mathrm{eV}$ for the treated ones with $\mathrm{BH}$ and BPA. From the integration of the peak area the modification with $\mathrm{BH}$ has larger surface density than BPA. implying that $\mathrm{BH}$ attachment on ITO surfaces is more efficient. Collectively. the above results confirm the immobilization of BDAs on the film surface. Although the electrochemical and XPS measurements support our idea that ITO electrode surfaces can be modified through the electrochemical manner. the nature of the linkages between primary amine and ITO surface and the exact mechanism of the oxidative attachment were not investigated in this stidy. However. we suppose that the mechanism and the resulting bond nature are similar to the corresponding ones on carbon and metal surfaces. Most plausible mechanism reported for carbon and metal surfaces is the formation of a radical cation through oxidation of the amine group. ${ }^{\text {? }}$

Next we applied the method to the patterning of biotinylated
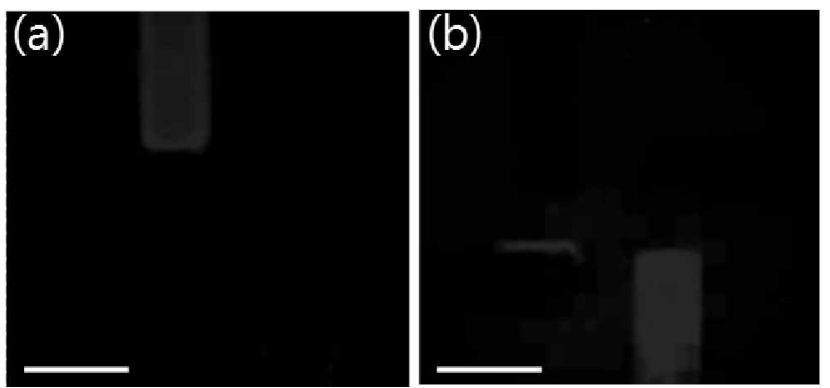

Figure 4. Fluorescence microscopic images after spatially selective and sequential immobilization of (a) Texasked-labeled and (b) FITC-labeled SA on BPH-modified IAIM. Scale bar $=200$ uㅡ.
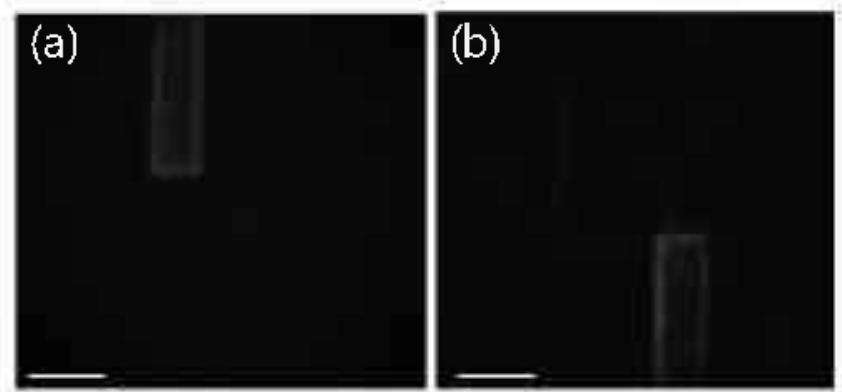

Figure 5. (a) Fluorescence microscopic image for the detection of rabbit IgG $(500 \mathrm{ng} / \mathrm{mL})$ resulted from the sandwich-type immunoassay based on the process in Scheme 1. After detecting rabbit IgG, the IAIM substrate was reused to detect molse IgG $(500 \mathrm{ng} / \mathrm{mL}$ ). The resulting image is shown in (b). Scale bar $=200$ $\mu \mathrm{m}$.

antibodies on the IAIM for the detection of antigens. where avidin (Av) or streptavidin (SA) was used as a linker molecule connecting biotinylated antibodies to BDA on the surfaces. The biospecific interaction between biotin and SA (association constants. $K_{\mathrm{c} 1} \sim 10^{15} \mathrm{M}^{-1}$ in solution) is very strong. ${ }^{2-}$ Prior to the use of antibodies. patterning of SA through the modification of the IAIM with BDAs was tested. BPH antong BDAs was employed for this modification because poly-ethyleneoxide (PEO) linkage inserted as a spacer between biotin and hydrazide group is known that it reduce nonspecific binding of protein on solid surfaces. ${ }^{2+}$ The procedure for the patteming of SA on the IAIM was as follows. First. BPH was selectively formed on an electrode of the array with the electrochemically directed manner. After washing with copious water the whole array surface presenting BPH-modified electrode was exposed to phosphate buffered saline (PBS) solution of $1 \mu \mathrm{g} / \mathrm{mL}$ SA labeled with TexasRed (red color) for 1 hour. After wasling the resulting surface with PBS and water. an opposite electrode of the same array was modified with $\mathrm{BPH}$ and 1 $\mu \mathrm{g} / \mathrm{nL}$ SA labeled with fluorescein isothiocyanate (FITC, green color) wia the same procedure as previously treated electrode. Figure 4 shows that both red and green fluorescent images on the same IAIM surface obtained by following the above procedures are highly contrasted. which indicates that the immobilization of BPH using our method is efficient for the attachment of SA and nonspecific binding of SA is negligible. 
We extended our method to the immunoassay. A procedure for the patterning and the detection is described in Scheme 1. We used two kinds of biotiny lated antibodies: anti-rabbit IgG and anti-mouse IgG. To save time for spending on sequential two steps. avidin immobilization followed by attachment of biotinylated antibody on the BPH-modified surface. Av complexes of each biotinylated antibody were used in the patterning. The complex was prepared from mixing a biotinylated antibody and Ay in the PBS solution followed by filtering with a centrifugal tube (cut-off MW: 60000 Dalton) at $14000 \times \mathrm{g}$ for $12 \mathrm{~min}$ to remove unreacted excess $\mathrm{Ar}$. Compared to the two steps. the use of the complex revealed that cross contamination of antibodies due to nonspecific binding was greatly decreased. which has been proven with fluorescence imaging in the immunoassay. This result is very important to give accurate data in the antigen detection without false positive. The decrease might be attributed to the removed interaction of a free biotinylated antibody (as shown in Scheme 1) and Av pre-attached surface. expected from the two steps approach. Figure 5 shows fluorescent images from the detection of two kinds of antigens based on the sandwich-type immunoassay. After detection of rabbit IgG by using anti-rabbit IgG labeled with tetramethyl rhodamine isothiocyanate (TRITC. red color). as shown in Figure 5a the resulting surface was reused to detect mouse IgG. Figure $5 \mathrm{~b}$ shows a detection result for mouse IgG. indicating that FITC-labeled anti-mouse IgG react exclusively with mouse IgG surface. Two differently colored detection images with ligh signal to noise ratio indicate that not only' cross reaction of two antigens is negligible but also the reusability of the sensor surface to detect another antigen can be achieved.

In conclusion we have demonstrated a simple method of electrochemically directed modification of ITO surfaces with primary amine-terminated molecules and its useful application for the preparation of platform for the sandwich-type immunoassy wa the micropatterning through sequential immobilization of two types of antibodies. It have been shown that the immunosensor platform gives a good result with high signal to noise ratio on the fluorescence image and negligible cross reaction and can be reused in the detection of another antigen. All advantages of our results might be attributed to the combination of two independent techniques for timesaving. The first is the electrochemically directed immobilization of BDAs and the second is the employment of $\mathrm{Av}$ complexes of antibodies.

\section{Experimental Section}

All of biotin-derivatized primary amines (BDAs) were purchased from Pierce (www.piercenet.com). Antigen antibody, and avidin (Av) were purchased from Sigma. and streptavidin (SA) was purchased from Fluka.

Phosphate buffered saline (PBS) solution consisted of 0.01 $\mathrm{M}$ phosphate and $0.15 \mathrm{M} \mathrm{NaCl}$. PBST is composed of PBS and $0.05 \%(w / w)$ tween-20. The antibody-Av complex was prepared from mixing a biotinylated antibody and $A v$ in the PBS solution followed by filtering with a centrifugal tube (cut-off MW: 60000 Dalton) at $14000 \times \mathrm{g}$ for $12 \mathrm{~min}$ to remove unreacted excess Av.
ITO electrodes array surface was cleaned successively with acetone, ethanol. and water. The surface was immersed in piranha solution for 5 seconds and then washed with pure water. After drying the substrate. the electrochemical modification of the aimed electrode on the array substrate was conducted in a cell with $0.05 \mathrm{M}$ BDA solution containing 0.1 $\mathrm{M} \mathrm{KCl}$. Afterward. the surface was washed with pure water and then inmersed in anti-rabbit IgG-Av complex solution for $30 \mathrm{~min}$. After washing of the resulting electrode surface with copious PBST, same procedure was repeated on the other electrode on the same surface with anti-mouse IgG-Ay complex.

After inmobilization of BDAs and the complexes. the surface was disclosed to a drop of antigen solution $(\mathrm{l} \mu \mathrm{g} / \mathrm{mL})$ and was incubated at room temperature for $\mathrm{l}$ hr. Following the washing, the surface was incubated again at room temperature with fluorescent labeled antibody solution $(1 \mu \mathrm{g} / \mathrm{mL})$ for $\mathrm{l} \mathrm{hr}$. After the washing and drying. the surface was subjected to the observation with an inverted fluorescent microscope (IX 71. Olynupus. Tokyo. Japan).

Acknowledgments. This work was supported by University of Incheon Research Grant in 2006.

\section{Refelences}

1. Manifacier, I. Thin Solid Films 1982, 90, 297.

2. Smith, D. L. Thin-film Deposition Principles \& Practice; McGraw-Hill: $1995 \%$ p 482.

3. Kim, Y. H.; Lee, S. T.; Jung, S. Y.; Bveon, K. N.; Kim, J. S.; Shin, S. C., Kwon, S. K. Bull Korean Chem. Soc. 2007, 28, 443.

4. Seok, W. K.; Gupta, A. K.: Roh, S.-T.; Lee, W.: Han, S.-H. Bull. Koreon Chem. Soc. 2007, 28, 1311 .

5. Gordon, R. G. IIRS Bull. $\mathbf{2 0 0 0}, 25,52$

6. Aziz, M. A.; Yang, H. Bull Korean Chem. Soc. 2007, 28, 1171

7. Heller, A.: Feldman, B. Chenical Reviews 2008, 108, 2482.

8. Bakker. E. Anal Chem 2004, 76, 3285.

9. Lee, T. M.-H.; Cai, H.; Hsing I.-M. Analyst 2005, 130, 364.

10. Pollack, M. G.; Fair, R. B.; Shenderov, A. D. Appl Phis. Lett. 2000, 77, 1725

11. O'Shannessy, D. T.: Brigham-Burke, M.: Peck, K. Anal Biochem. $1992,205,132$

12. Gardner, T. J.; Frisbie, C. D.; Wrighton, M. S. J. Ant Chent Soc: $1995,117,6927$

13. Oh. S.-Y.: Yun, Y.-J.: Kim, D.-Y.: Han, S.-H. Langmii 1999. 15,4690 .

14. Kim, K, Song, H.; Park, J. T.; Kwak, J. Chem. Lett. $2000,958$.

15. Napier, M. E.: Thor, H. H. Langmii $1997,13,6342$.

16. Das, J.: Aziz, M. A.: Yang, H. J. Am. Chem. Soc. 2006, 128, 16022 .

17. Kang, M. S.; Ma, H.; Yip, H. L.; Jen, A. K. Y. J. Moter. Chem. $\mathbf{2 0 0 7}, 17,3489$

18. Maldonado, S.: Smith. T. J.: Williams, R. D.: Morin, S.: Barton, E.: Stevenson, K. J. Langmir 2006, 22, 2884.

19. Deinhammer, R. S.; Ho, M; Anderegg, J. W; Porter, M. D. Langmitr 1994, 10, 1306.

20. Adenier, A.; Chehimi, M. M.: Gallardo, I.; Pinson, I.; Vilä, N. Langmir 2004, 20,8243.

21. Kim, E. J;: Shin, H. Y.; Park, S.; Sung, D ; Jon, S.; Sampathkumar, S. G.; Yarema, K. I.; Choi, S. Y.; Kim, K. Chem. Conmmu. 2008, 3543.

22. Kim, K, Kwak, T. J. Electroanal. Chem. 2001, 83, 512

23. Kirley, T. L. Anal Biochem. 1989, 10, 231.

24. Prime, K. L.; Whitesides, G. M. I. Ant. Chent. Soc. 1993, 115, 10714. 